\section{Schmerz und Alter}

Bei den Alten und den Kleinsten werden Schmerzen immer noch vernachlässigt.

Die Erkenntnis, dass kognitive Einschränkungen, etwa bei älteren Menschen mit Demenz, nicht vor Schmerzen schützen, setzt sich nur mühsam durch (siehe auch den Beitrag „Der alte Mensch als Schmerzpatient", Seite 51 ff. in diesem Heft). Die Deutsche Akade-

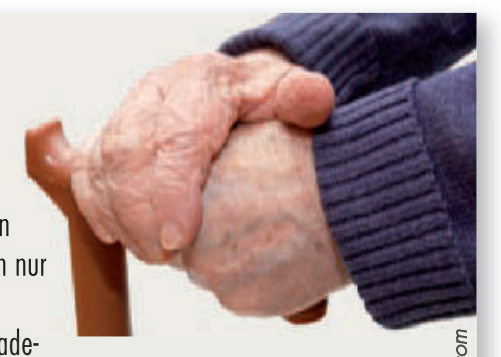
mie für Ganzheitliche Schmerztherapie e.V. (DAGST) erinnert daran, dass auch der Irrglaube, Säuglinge und Kleinkinder würden keine Schmerzen empfinden, immer noch weit verbreitet ist (vgl. die beiden Beiträge „Die Schmerzwahrnehmung beginnt schon vor der Geburt" [ASUP 3/2008, Seite 26 ff.] und "Was hilft wirklich gegen Schmerzen bei Kindern?" [ASUP 4/2008, Seite 33 ff.] von Dr. Sven Gottschling). Schmerz wird in der Gesellschaft als unvermeidbarer Teil des Alterns angesehen: „Wer über fünzzig ist und morgens ohne Schmerzen aufwacht, ist tot." Und über Säuglinge und Kleinkinder kann man immer noch hören: „Die sind so klein, die schreien immer und haben noch keine Schmerzen."

Elementar sind daher eine differenzierte Herangehensweise und genaue Schmerzerfassung bei Patienten, die nur eingeschränkt kommunikationsfähig sind, weil sie entweder zu klein oder zu alt und krank sind. Dies will die DAGST in der Veranstaltung "Schmerz und Alter" am 27. November in Köln vermitteln. Berücksichtigt werden sowohl die Belange der Säuglinge und Kleinkinder als auch die der alten, hochbetagten und dementen Menschen in Einrichtungen der Altenhilfe und Altenpflege.

Die zunehmende Verbreitung von Leitlinien, die krankheitsbezogen entwickelt werden, sich aber auf Menschen mit vielen Erkrankungen nur schwer umsetzen lassen, macht das Herangehen in der Praxis schwierig. Für Säuglinge und Kleinkinder ist das umgekehrte Phänomen feststellbar: Es gibt kaum Leit-

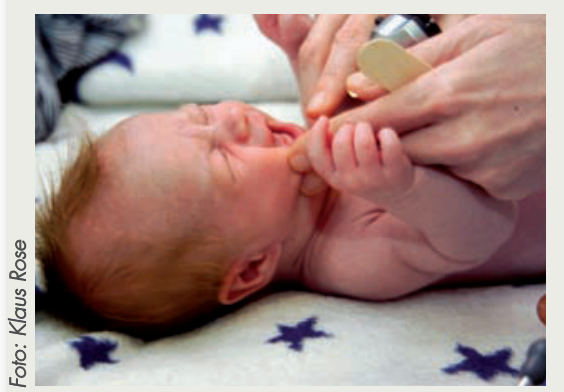
linien, und die meisten Medikamente müssen "off |abel" eingesetzt werden, was teilweise zu großer Verunsicherung und damit zu Unterversorgung führt. Deshalb zeigt die Veranstaltung auch den sinnvollen Einsatz und die Möglichkeiten von komplementären Verfahren in diesen Altersgruppen auf. In den angebotenen Workshops können die gewonnenen Erkenntnisse vertieft und angewendet werden.

Weitere Informationen auf der Homepage der DAGST (www.dagst.de) sowie auf Seite 50 in diesem Heft.

Daher fordert die Koalition gegen den Schmerz, dass Opioide der WHO-Stufe III aus der automatischen Austauschpflicht nach §129 SGB $\vee$ herausgenommen werden und nicht vom Apotheker ohne Rücksprache mit dem verordnenden Arzt ausgetauscht werden dürfen - selbst dann nicht, wenn kein Aut-idem-Kreuz gesetz $\dagger$ wurde.

\section{Schmerztherapie in der Approbationsordnung verankern}

Darüber hinaus kritisierte Prof. Dr. RolfDetlef Treede, Mannheim/Heidelberg, den gravierenden Mangel an Ärzten in Deutschland, die für die Schmerztherapie qualifiziert sind: „Wir können es uns nicht leisten, dass Patienten über Jahre von einem Arzł zum nächsten gehen, weil ihre Schmerzen nicht adäquat diagnostiziert und behandelt werden", sagte der Präsident der Deutschen Gesellschaft zum Studium des Schmerzes e.V. (DGSS). Die Schmerzforschung sei in Deutschland weltweit Spitze, doch die Erkenntnisse würden nicht bei den Studenten, den Ärzten und den Patienten ankommen, mahnte auch Müller-Schwefe.

Aus diesem Grund tritt die Koalition gegen den Schmerz zudem dafür ein, die Schmerztherapie als Querschnittsfach fest in der Approbationsordnung für Ärzte zu verankern. den Schmerz' an die Gesundheitspolitik - Schmerzpatienten besser versorgen - Ärzte besser ausbilden, Berlin, 20. Mai. 2010; Veranstalter: Koalition gegen den Schmerz
Pressegespräch „Aktuelle Forderungen der ,Koalition gegen

\section{Heftige Reaktionen auf ein BGH-Urteil}

Das Urteil des Bundesgerichtshofs $(B G H)$ vom 25. Juni zum Fall „Erika K." hat ein intensives Echo ausgelöst. Das Spektrum der Reaktionen reicht von Erleichterung bis Entsetzen.

In einer offiziellen Mitteilung des BGH heißt es, dass es nicht darauf ankomme, ob der Abbruch [einer Behandlung] durch aktive Handlungen erfolgt, also beispielsweise durch das Durchtrennen einer Magensonde. Die Einwilligung der Patientin "rechtfertigte nicht nur den Behandlungsabbruch durch bloßes Unterlassen weiterer Ernährung, sondern auch ein aktives Tun, das der Beendigung oder Verhinderung einer von ihr nicht oder nicht mehr gewollten Behandlung diente."

Unmittelbar nach Bekanntwerden des Urteils nahm die "Deutsche Hospiz Stiftung" Stellung: „Wir sollten uns im Klaren darüber sein, dass nicht alles, was straflos bleibt, auch geboten ist", kommentierte der Geschäftsführende Vorstand, Eugen Brysch. Das Urteil sende nach seinen Worten ein fatales Signal aus, das dem Grundrecht Schwerstkranker auf Selbstbestimmung und Fürsorge nicht gerecht werde; es sei zu befürchten, dass dem Missbrauch Tür und Tor geöffnet werde.

Im Gegensatz dazu hat der "Deutsche Hospiz- und PalliativVerband" (DHPV) das Urteil des BGH begrüßt und sieht darin eine Stärkung der Selbstbestimmung schwerstkranker und sterbender Menschen. Das Urteil stelle klar, dass eine Behandlung nicht gegen den Willen der Betroffenen durchgeführt werden darf. Das sorge für mehr Rechtssicherheit.

Der DHPV widerspricht damit der oben zitierten Erklärung der "Deutschen Hospiz Stiftung", die nach eigenem Bekunden weder Dachverband noch Interessenvertretung hospizlicher Einrichtungen sei und ihre bisher geleistete Förderung für ambulante Hospizdienste im Dezember 2009 eingestellt habe. Allerdings sieht auch der DHPV Handlungsbedarf, etwa wenn es darum gehe, dass die Voraussetzungen zur Wahrnehmung des Selbstbestimmungsrechts verbessert werden. 